\title{
О ДИНАМИКЕ КВАЗИКАНОНИЧЕСКИХ СИСТЕМ
}

I. KEIS. KVAASIKANOONILISTE SUSTEEMIDE DONAAMIKAST

1. KEIS. ON THE DYNAMICS OF THE QUASI-CANONICAL SYSTEMS

(Представил Н. Алумяэ)

В работе, продолжающей исследования $\left[{ }^{1-3}\right]$, рассмотрен ряд интегральных и локальных свойств канонизируемых систем (квазиканонических). Эти системы заданы союзными с $\omega(d x, d t) \triangleq b \cdot d x+h d t$ уравнениями

$$
\begin{gathered}
R x=a, R=\left[r_{i \sigma}\right], r_{i \sigma}=\partial b_{i} / \partial x_{\sigma}-\partial b_{\sigma} / \partial x_{i}, x=d x / d t \quad(i, j, \sigma=\overline{1,2 n}), \\
a=\nabla_{x} h-\partial b / \partial t, \quad b=\left(b_{j}\right)^{*}, \\
\quad x=\left(q_{1}, \ldots, q_{n}, p_{1}, \ldots, p_{n}\right)^{*}, \Delta R \stackrel{\Delta}{=} \operatorname{det} R>0 \quad\left(a \cdot b=\sum_{i=1}^{2 n} a_{j} b_{j}\right),
\end{gathered}
$$

где форма $\omega-$ класса $2 n+1 \rightarrow(\Delta R>0), b(t, x), h(t, x)-$ достаточно гладкие функции. Гомеоморфизм $t=\vartheta(t, x), \widetilde{x}=f(t, x)$ переводит (1) в квазиканоническую систему, союзную с $\tilde{\omega}$, полученной из $\omega$ гладким отображением $t \rightarrow \tilde{t}, x \rightarrow \tilde{x}$. В нормальных переменных $[4,5]$ формы $\omega(d x, d t): \tau=\tau(t, x), \xi=\xi(t, x)$ имеем $\omega \triangleq P \cdot d Q-G(\tau, Q, P) d \tau$, и система (1) переходит в гамильтонову $\left[{ }^{4}\right]$

$d \xi / d \tau=Z \nabla_{\xi} G, Z=\left[\begin{array}{cc}-0, & \mathbf{1}_{n} \\ -\mathbf{1}_{n}, & 0\end{array}\right]$ при $c_{1} \leqslant \vartheta ; \quad \tau \leqslant c_{2}<\infty, c_{1}>0, c_{1,2}=$ const.

Всякая квазиканоническая система является скрытой канонической.

Итегральные свойства. Существование инварианта $I_{1}^{0}=\oint_{b} \cdot \delta x$ $\left(\Omega^{0} \stackrel{\Delta}{=} b \cdot d x\right.$ - класса $\left.2 n\left[{ }^{4,5}\right]\right)-$ критерий квазиканоничности, т. е. представимости системы в виде (1) с $\Delta R>0\left(\forall h \subset C_{1}(t, x)\right)$.

Аналогично $\left[{ }^{6}\right]$, любой линейный по $\delta x$ относительный инвариант $\oint b^{*} \cdot \delta x \stackrel{\Delta}{=} I_{1}^{*} \equiv c^{*} I_{1}^{0} \quad$ системы (1), т.е. $\Omega^{*}=c^{*} \Omega^{0}$, где $c^{*}=$ const. Система (1) имеет абсолютный инвариант порядка $2 n$ и вида

$$
\begin{gathered}
I_{2 n}=\int_{\Omega} M(t, x) \delta x_{1} \ldots \delta x_{2 n}, \\
M+M \operatorname{div}_{x} \cdot X(t, x)=0\left(X=x, \text { здесь } x=R^{-1} a\right) .
\end{gathered}
$$

Из (1), (3) в новых независимых переменных $\theta=\theta(t, x), y=y(t, x)$ находим

$$
d y / d \theta=\tilde{\lambda}^{-1} Y(\theta, y), \quad d N / d \theta+N(\theta, y) \operatorname{div}_{y}\left(\tilde{\lambda}^{-1} Y\right)=0
$$




$$
\left(N=N(\theta, y), \tilde{\lambda}(\theta, y)=\theta^{*}\right)
$$

и формулу связи множителей $M, N$ исходной и преобразованной системы (4)

$N=\tilde{\lambda} M \tilde{\theta}_{t}^{-1} \Delta^{-1}(J), \quad J=[\partial y / \partial x], \quad \tilde{\theta}_{t}=\lambda^{*}\left(0, y_{0}\right)+\int_{0}^{t} \lambda_{t}^{*} d \sigma \quad\left(\theta^{*}=\lambda(t, x)\right)$.

При известных функциях $\tilde{\tau}_{t}, \Delta\left(J^{0}\right)$ от $t, x$ и свойстве $N^{0}=1$ системы (2) получим из (5) значение $M$ в (3)

$$
\begin{gathered}
M(t, x)=\lambda^{-1} \tilde{\tau}_{t} \Delta\left(J^{0}\right), \quad \lambda=\lambda(t, x)=d \tau / d t, \quad J^{0}=[\partial \xi / \partial x] \quad(\theta \rightarrow \tau), \\
\tilde{\theta}(t, y) \stackrel{\Delta}{=} \theta(t, x(t, y)), \quad \tilde{\tau}(t, \xi)=\tau(t, x(t, \xi)), \quad \lambda^{*}(t, y)=\lambda[t, x(t, y)] .
\end{gathered}
$$

Рассмотрим стационарный на $\dot{x}(t)$-решениях (1). функционал Пфаффа

$$
I[\tilde{x}]=\int_{t_{0}}^{t}(b \cdot \tilde{x}+h) d \sigma, \quad \delta I[x]=0
$$

при фиксированных концах $t_{0}, t, \tilde{x}\left(t_{0}\right), x(t)$. Так как (1) удовлетворяет модифицированному принципу Ливенса [ $\left.{ }^{4}\right]$, то она имеет на $x(t)$ вариационную форму $\delta[L]_{x}=0, \quad$ где $L=b \cdot \tilde{x}+h, \quad \mathcal{E}=\nabla_{\tilde{x}}-d / d t \circ \nabla \tilde{x}$. Отсюда при $b_{t}=0, h_{t}=0$ и $b_{x j}=0, h_{x j}=0$ система (1) имеет соответственно интегралы $h=h_{0}=$ const и $b_{j}=b_{j 0}=$ const.

Локальны е с вой ства. Обозначим $I[x]=S\left(t, t_{0}, x_{0}\right)$. В случае свободных концов найдем, учитывая (6), вариацию $\delta I[x]$ в виде

$\delta \tilde{S}=\left.(b \cdot \delta x+h \delta t)\right|_{t_{0}} ^{t}, \quad \partial \widetilde{S} / \partial t=-h\left(t_{0}, x_{0}\right)=-h_{0}, \quad d S\left(t, x_{0}\right) \stackrel{\Delta}{=} \delta \widetilde{S}+h_{0} \delta t$.

Фиксируя $t_{0}$ в (7), получим, что определенное (1) преобразование $x_{0} \rightarrow x, t_{0} \rightarrow t, x=x\left(t, x_{0}\right)$ имеет локальное свойство, данное уравнением

$$
b(t, x) \cdot d x+h(t, x) d t=b\left(t_{0}, x_{0}\right) d x_{0}+d S\left(t, x_{0}\right) \quad\left(x=x\left(t, x_{0}\right)\right) .
$$

Справедливо обратное: если в переменных

$$
t, \gamma: \omega=k\left(t_{0}, \gamma\right) \cdot d \gamma+d K(t, \gamma),
$$

$x=\tilde{x}(t, \gamma), \Delta[\partial \widetilde{x} / \partial \gamma] \neq 0, \gamma=\gamma\left(x_{0}\right)$, то $\tilde{x}(t, \gamma)$ - решения (1).

Объединяя утверждения (8), (9), имеем для (1) модификацию теоремы эквивалентности $\left[{ }^{4}\right]$. Найдем в переменных $t, x$ представление $\mathcal{C}$-канонического преобразования эквивалентной (1) системы (2). Определим $\mathcal{C}$ равенствами

$$
\begin{gathered}
b\left(t^{\prime \prime}, x^{\prime \prime}\right) \cdot d x^{\prime \prime}+h\left(t^{\prime \prime}, x^{\prime \prime}\right) d t \equiv c^{0}\left[b\left(t^{\prime}, x^{\prime}\right) \cdot d x+h\left(t^{\prime}, x^{\prime}\right) d t^{\prime}\right]-H_{0} d \tau-d \Phi, \\
\tau=\tau(t, x), \quad \xi=\xi(t, x), \quad c^{0}=\mathrm{const} \neq 0, \quad \begin{array}{c}
\tau\left(t^{\prime}, x^{\prime}\right)=\tau\left(t^{\prime \prime}, x^{\prime \prime}\right)=\tau, \\
\forall t^{\prime \prime}=\theta\left(t^{\prime}, x^{\prime}\right), \quad x^{\prime \prime}=y\left(t^{\prime}, x^{\prime}\right),
\end{array}
\end{gathered}
$$

где $\tau, \xi-$ канонические переменные $\omega\left[{ }^{5}\right], H_{0}, \Phi-$ произвольные функции. Действительно, переходя в тождестве (10) от $t, x \mathrm{k} \tau$,, , получим достаточное условие каноничности преобразования $\tau^{\prime} \rightarrow \tau^{\prime \prime}=\tau=\tau^{\prime}$, $\xi^{\prime} \rightarrow \xi^{\prime \prime}$ вида

$P^{\prime \prime} \cdot d Q^{\prime \prime}=c^{0} P^{\prime} \cdot d Q^{\prime}-H^{0} d \tau-d \Phi^{0} \quad\left(H^{0}=H_{0}+c_{0}-1, \quad \Phi^{0}\left(\tau, \xi^{\prime}, \xi^{\prime \prime}\right)=\Phi\right)$

Частным случаем асинхронного преобразования (10) будет обобщенно- 
ка̇ноническое $\hat{C}^{0}$-преобразование (11), сохраняющее каноничность в новом времени $\tau^{\prime}$ :

$p^{\prime} \cdot d q^{\prime}-H^{\prime} d \tau^{\prime}=c^{0}(p \cdot d q-H d \tau)-d F \quad\left(\tau^{\prime}=T(\tau, q, p), \xi^{\prime}=y(\tau, q, p)\right.$.

При $c_{0}=1, H_{0}=0, \Phi_{0}=-\widetilde{S}$ из. (7), (10) находим свойство (8) решения $x\left(t, x_{0}\right)$ системы (1). Поэтому эквивалентная (1) каноническая система (2) переходит на преобразовании $t_{0} \rightarrow t, x_{0} \rightarrow x\left(t, x_{0}\right)$ в каноническую систему.

$\mathrm{B}\left[{ }^{3}\right]$ отмечено, что любая линейная неголономная или оптимальная управляемая динамическая система с интегральным инвариантом типа Пуанкаре-Картана является скрытой канонической. Отсюда следует, что она будет квазиканонической системой (1), обладающей инвариантом $I_{1}{ }^{0}$ и динамическими свойствами $(3),(6),(8),(9)$.

Пример. Двумерная неавтономная (или автономная) система Лагранжа

$q=H_{p}, \quad p^{\cdot}=-H_{q}+f(t, q, p) \quad\left(H=H(t, q, p), \quad \partial^{2} H / \partial p^{2} \neq 0, \quad \Phi_{x} \stackrel{\triangle}{=} \partial \Phi / \partial x\right)$

есть квазиканоническая, ибо существуют решения $b_{1}, b_{2}, h$ системы

$$
h_{p}=\varrho H_{p}+b_{2 t}, \quad h_{q}=\varrho\left(H_{q}-f\right)+b_{1 t} \quad\left(\varrho \stackrel{\Delta}{=} b_{2 q}-b_{1 p}, \Delta R=\varrho^{2}\right)
$$

при условии, что форма $\omega^{0}=b_{1} d q+b_{2} d p+h d t-$ класса 3. Это эквивалентно

$$
\varrho \neq 0, h \neq\left(H_{q}-f\right) b_{2}-H_{p} b_{1}\left(\sim \omega^{0} \neq 0 \text { на }(12)\right) .
$$

Система (13) совместна лишь тогда, когда $\mathrm{Q}=M-$ множитель (12), т. е. $M \cdot=-M f_{p}$. Решения, удовлетворяющие (14), имеют вид

$$
\begin{gathered}
\varrho=\varrho^{0}\left(q_{0}, p_{0}\right)\left|\partial(q, p) / \partial\left(q_{0}, p_{0}\right)\right|^{-1}, \quad b_{1}=b_{1}^{0}(t, q, p), \\
b_{2}=\int_{q_{0}}^{q}\left(b_{1 p}+\varrho\right) d q+b_{2}^{0}(t, p), \\
h=\int_{q_{0}}^{q}\left[\varrho\left(H_{q}-f\right)+b_{1 t}\right] d q+\left.\int_{p_{0}}^{p}\left[\varrho H_{p}+b_{2 t}\right]\right|_{q=q_{0}} d p+h^{0}(t),
\end{gathered}
$$

где произвол функций $\mathrm{Q}^{0}, b_{1}{ }^{0}, b_{2}{ }^{0}, h^{0}$ отмеченных аргументов ограничен условием (14).

\section{ЛИТЕРА Т У РА}

1. Ча плыгин С. А., Исследования по динамике неголономных систем, М-Л., ГИТТЛ, 1949 , с. $28-38$.

2. Биркгоф Дж. Д., Динамические системы, М.-Л., ОГИЗ, ГИттЛ, 1941, c. $34-54$.

3. Ке й с И., Изв. АН ЭССР, Физ. Матем., 30, № 2, 109-116 (1981).

4. П а р с Л., Аналитическая динамика, М., «Наука», 1971, с. 286-289, 410-418, $531-532$.

5. Р а ше в ск и й П. К., Геометрическая теория уравнений с частными производными, М.-Л., ОГИЗ, Гостехиздат, 1947, с. $142-168$.

6. Hwa-Chung Lee, Proc. Roy. Soc. Edinburgh, ser. A, LXII, 237-247 (1947).

Институт кибернетики

Академии наук Эстонской ССР
Поступила в редакцию 30/XII 1981 